
\title{
25 Research Soure \\ Risk Occurred Probability and Influencing Factors of Stroke in Followed-Up Hypertension Patients
}

\section{An-le Li ( $\square$ anle_li@aliyun.com )}

Jiading district center for disease control and prevention, Shanghai, China

Ying Qi

Jiading district center for disease control and prevention, Shanghai, China

Shuai Zhu

Jiading district center for disease control and prevention, Shanghai, China

Zhi-hao Hu

Jiading district center for disease control and prevention, Shanghai, China

Xuejin Xu

Jiading district center for disease control and prevention, Shanghai, China

Ya-wei Wang

Jiading district center for disease control and prevention, Shanghai, China

Xin-zhi Qian

Jiading district center for disease control and prevention, Shanghai, China

\section{Research Article}

Keywords: Hypertension, Stroke, Risk probability, Factors, Cox regression, Cohort

Posted Date: August 20th, 2021

DOI: https://doi.org/10.21203/rs.3.rs-760348/v1

License: (c) (i) This work is licensed under a Creative Commons Attribution 4.0 International License.

Read Full License 


\section{Abstract \\ Objective}

To explore the risk occurred probability and influencing factors of stroke in followed-up hypertension (HP) patients through the analysis of long-term followed-up cohort data.

\section{Methods}

The method of followed-up observation cohort was used to collect the information of 168417 followedup hypertension patients from 2002 to 2020 in Jiading district in Shanghai. Kaplan-Meier method was used to analyze the risk occurred probability of stroke complications in long-term followed-up HP patients, and the influencing factors were analyzed by Cox proportional risk model.

\section{Results}

Among 168417 followed-up hypertension patients, 11143 cases had occurred stroke, and the cumulative occurrence rate of stroke was $6.62 \%$ (male was $6.87 \%$, female was $6.37 \%$ ). With the extension of the years of hypertension, the cumulative risk occurred probability of stroke in HP patients would continue to increasing, and the interval was not equidistant. The total cumulative risk probability of stroke in HP patients was $78.9 \%$ (male was $91.0 \%$, female was $70.7 \%$ ). During the period of hypertension, the risk occurred probability of stroke was not fixed, but fluctuates. There were 4 onset peaks, which were in 8 years (peak value 4.2\%), 15 years (peak value 14.0\%), 22 years (peak value 6.0\%) and 26 years (peak value $13.9 \%$ ). The highest risk probability of male patients was in 26 years (peak value $23.1 \%$ ), and the second peak was in 15 years (peak value 15.6\%). The highest risk probability of female patients was in 15 years (peak value 12.9\%), and the second peak was in 26 years (peak value $8.7 \%$ ). The risk probability of different sex, BP grad and BMI was different, male was higher than female, grade 3 HP higher than grade 1 and grad $2 \mathrm{HP}$, thin higher than normal weight. The major influencing factors of stroke complications were age $(R R=2.917, p<0.001)$, body mass index $(R R=1.450, p<0.001)$, family history of stroke $(R R=1.386, p<0.001)$, blood pressure grad $(R R=1.148, p<0.001)$, registry age $(R R=1.071, P<$ 0.001 and family history of hypertension $(R R=1.051, P<0.001)$.

\section{Conclusion}

The risk occurred probability of stroke among hypertension would continue to increase disproportionately during period of hypertension, and the total cumulative risk probability could finally reach $78.9 \%$. Male was higher than female. Age, BMI, family history (stroke and HP), blood pressure grad and duration of HP were related to the occurrence of stroke complications. 


\section{Introduction}

Stroke is an acute cerebrovascular disease caused by many factors, and it is the first cause of death in China and the second largest cause of death in the world [1-3]. Hypertension, diabetes, dyslipidemia, cardiovascular disease and unhealthy lifestyle are all risk factors of stroke, among which hypertension is recognized as the main modifiable risk factor of stroke [4-6]. The guidelines of the American Stroke Association also believe that controlling the risk factors of stroke is an effective prevention and control strategy for stroke [7]. Hypertension patients were prone to falling stroke, but not all hypertension patients would have stroke, and previous community population observation results showed that different hypertension patients have different epidemic characteristics and prevalence probability of stroke. In the followed-up population of hypertension patients, ischemic stroke was the most important type, rather than hemorrhagic stroke [8].

In order to better observe and explore the risk probability and risk factors of stroke in hypertension patients, the data of a long-term follow-up cohort of hypertensive patients in Jiading district in Shanghai was used in this study, and the risk probability and influencing factors of stroke in followed-up hypertension patients was analyzed and explored. We hope that these results can provide some help for the prevention and control of stroke in community in the future. The Follow-up Management System of hypertension have been formally established and implemented in Jiading district in Shanghai China since from 2002. The Hypertension Follow-up Management System Database records the medical information of all registered and followed-up hypertension patients. According to the guidelines and policies of hypertension prevention and control, the hypertension patients in communities were registered and followed up, family doctors and public health personnel in the community health service center are responsible for the registration, follow-up and management of hypertension patients in the community, the center for disease control and prevention is responsible for the formulation of technical scheme, the training of personnel and the quality control of follow-up data. All patients were essential hypertension. All medical followed-up information of patients must be truthfully recorded in the system database by follow-up doctors.

\section{Methods}

\section{Data sources}

All patients were from the Hypertension Follow-up Management System in Jiading district in Shanghai China, and the system database recorded the medical information of all registered and followed-up hypertension patients. The hypertension patients in community were registered, investigated, filed and followed-up regularly by community health workers or family doctors. A total of 168417 hypertension patients were entered into the database of the observation as the deadline of September 30, 2020. All about stroke occurred in followed-up patients must be recorded in detail. Stroke must be diagnosed and

confirmed by a senior hospital. This study was approved by Science and Technology Commission, Health 
Commission and CDC in Jiading district in Shanghai. All methods were carried out in accordance with relevant guidelines and regulations.

\section{Data Collection}

The registered information and followed-up records of hypertension patients were recorded in the system, and collected from the system mainly include the date of birth, gender, ID number, residential address, occupation, education level, family history, date of establishment, termination date and reasons for termination, blood pressure and blood pressure at each follow-up, history of stroke (type of stroke, diagnostic date and diagnostic hospital), lifestyle (smoking, drinking and physical activity) and so on.

The date of occurrence of stroke in hypertension patients must be later than the date of registration. If the date of onset of stroke was earlier than or close to the date of registration, this patient data would not be included.

\section{Definition And Classification}

The diagnosis of stroke was based on the clinical diagnostic criteria (corresponding clinical symptoms plus positive results of head CT or MRI) $[9,10,11]$. The specific diagnosis was made by the secondary and tertiary hospitals, and then the relevant information was collected by family doctors in the community. Stroke mainly included ischemic stroke, hemorrhagic stroke and unclassified stroke in this study. Ischemic stroke include transient ischemic attack, cerebral infarction. Hemorrhagic stroke included subarachnoid hemorrhage, intracerebral hemorrhage and other non-traumatic intracranial hemorrhage.

The measurement and classification of blood pressure and the diagnosis of hypertension were carried out according to the standard of Chinese guidelines for the prevention and treatment of hypertension [11-13]. The diagnosis of hypertension was implemented by advanced clinical hospitals. The hierarchical management of hypertension patients was divided into high-risk, medium risk and low-risk groups according to the blood pressure value, clinical symptoms, exposure risk factors, clinical complications and target organ damage of patients. The follow-up management requirements of different groups are different.

Observation starting point refers to the time point when hypertension patients were registered and entered the follow-up management queue. Observation end point refers to the time when hypertension patients have expected outcome events (stroke) or withdraw from the observation queue due to loss of follow-up. Follow up time or observation duration refers to the time difference between the end point and the starting point (observation end point minus observation starting point). Censored values were calculated for all study populations that did not reach the observed end point.

\section{Statistical analysis}


According to the deadline, all the recorded data in the hypertension follow-up management system before the deadline were exported to the Microsoft Excel database, and then the corresponding logic check, data screening and conversion were carried out. Finally, the sorted database was imported into the SPSS statistics software package (IBM SPSS statistics version 21) for statistical analysis. The mean and standard deviation of age, blood pressure and other quantitative data were calculated. The number and frequency (\%) were calculated according to gender, number of patients, etc. The chi-square $\left(\mathrm{X}^{2}\right)$ test was used to compare the data between different groups. The risk probability was analyzed by Kaplan-Meier method, and the related influencing factors were analyzed by Cox regression. Bilateral test, $\mathrm{P}<0.05$ for the difference was statistically significant.

\section{Results}

\section{Baseline demographic characteristics}

In this study, 11143 cases had developed stroke before the deadline among 168417 followed-up hypertension patients in Jiading district in Shanghai China. The complicated ratio of stroke among followed-up hypertension patients was $6.62 \%$. Among them, 5636 cases of stroke occurred in male hypertension patients, and the complicated ratio of stroke was $6.87 \%$. There were 5507 cases of stroke in female hypertension patients, and the complicated ratio of stroke was $6.37 \%$. Male was higher than female $\left(x^{2}=17.091, p<0.001\right)$.

Among these observational hypertension patients, no stroke occurred in patients under 30 years old (the complicated ratio was 0 ), 21 cases of stroke occurred among the patients aged $30 \sim 39$ years old (the complicated ratio was $0.68 \%$ ), 250 cases of stroke occurred among the patients aged $40 \sim 49$ years old (the complicated ratio was 1.70\%), 1417 cases of stroke occurred among the patients aged $50 \sim 59$ years old (the complicated ratio was 3.29\%), 3629 cases of stroke occurred among the patients aged $60 \sim 69$ years old (the complicated ratio was 5.93\%), 3800 cases of stroke occurred among the patients aged 70 $\sim 79$ years old (the complicated ratio was $11.79 \%$ ), 2026 cases of stroke occurred among the patients aged 80 years old and above (the complicated ratio was 14.63\%). The older the patients, the higher complicated ratio of stroke $\left(x^{2}=424.276, p<0.001\right)$.

2118 cases of stroke occurred among smoking patients (the complicated ratio was 5.97\%), 255 cases of stroke occurred among occasionally smoking patients (the complicated ratio was $5.21 \%$ ), 976 cases of stroke occurred among patients who used to smoke but now don't smoke (the complicated ratio was 9.92\%), 7794 cases of stroke occurred among never smoking patients (the complicated ratio was 6.59\%). There were significant differences in the complicated ratio of stroke among different smoking patients $\left(x^{2}=213.188, p<0.001\right)$.

8966 cases of stroke occurred among never drinking patients (the complicated ratio was7.00\%), 1353 cases of stroke occurred among occasionally drinking patients (the complicated ratio was $5.40 \%$ ), 622 cases of stroke occurred among frequently drinking patients (the complicated ratio was 6.04\%), 222 
cases of stroke occurred among patients who drink every day (the complicated ratio was $4.04 \%$ ). There were significant differences in the complicated ratio of stroke among different drinking patients $\left(\mathrm{x}^{2}=\right.$ $149.764, p<0.001)$.

4534 cases of stroke occurred among normal weight patients (the complicated ratio was $5.30 \%$ ), 316 cases of stroke occurred among thin patients (the complicated ratio was 4.66\%), 6293 cases of stroke occurred among overweight or obese patients (the complicated ratio was $18.46 \%$ ). There were significant differences in the complicated ratio of stroke among different body mass index patients $\left(x^{2}=149.764, p\right.$ $<0.001)$.

\section{Risk Probability Of Stroke In Hp Patients}

To explore the change of risk occurred probability of stroke in hypertension patients during period of hypertension, the hypertension time (unit is year) was selected as the horizontal axis of time, and the occurrence of stroke (including ischemic stroke, hemorrhagic stroke and unclassified stroke) was defined as the event state, and Kaplan-Meier method was used to analyze the risk occurred probability of stroke. The calculated risk probability of each time point was made as a longitudinal axis and a dynamic curve was drawn. See Fig. 1. The result of Fig. 1 showed that the risk probability of stroke in HP patients was not fixed during the whole hypertension period, but fluctuates. There were 4 peak onset periods, which were in 8 years (peak value 0.042, 4.2\%), 15 years (peak value 0.140, 14.0\%), 22 years (peak value 0.060, $6.0 \%$ ) and 26 years (peak value $0.139,13.9 \%$ ). The highest risk of male patients was in 26 years (peak value $0.231,23.1 \%$ ), and the second peak was in 15 years (peak value $0.156,15.6 \%$ ). The highest risk of female patients was in 15 years (peak value $0.129,12.9 \%$ ), and the second peak was in 26 years (peak value $0.087,8.7 \%)$.

If cumulative occurred probability was taken as the vertical axis (see Fig. 2), the result showed that cumulative risk occurred probability of stroke in male and female patients would continue to increase with the time extension of hypertension years, and the proportion of increase was not equidistant. The occurred probability was higher in men than that in women $\left(x^{2}=113.570, p<0.001\right)$. The total cumulative risk probability of stroke in hypertension patients was 0.789 (78.9\%), and male was 0.910 (91.0\%), female was $0.707(70.7 \%)$.

The result of Fig. 3 showed that the cumulative risk probability of stroke in different blood pressure (BP) patients would continue to increase with HP time. The occurred probability was obviously higher in grad 3 (SBP $\geq 180 \mathrm{mmHg}$ or/andDBP $\geq 110 \mathrm{mmHg}$ ) patients than that in grad 2 (SBP:160

179mmHgor/andDBP:100 109mmHg) and grad 1 (SBP:140 159mmHg or/and DBP:90 99mmHg) patients and $\left(x^{2}=189.139, p<0.001\right)$.

Figure 4 showed that the cumulative risk probability of stroke in different body mass index (BMI) patients would continue to increase with hypertension time. The risk probability was higher in thin $(\mathrm{BMI}<18.5)$ patients than that in normal weight (BMI: $18.5 \sim 23.9)$ and overweight $(B M I \geq 24.0)$ patients $\left(x^{2}=\right.$ 
$136.724, p<0.001)$. The risk probability of stroke in normal weight patients would suddenly increase after hypertension 22 years and so on, and the reason was unknown.

The result of Fig. 5 showed that the cumulative risk probability of stroke in different family history $(\mathrm{FH})$ patients would continue to increase with hypertension time. But the occurred probability wasno difference between hypertension patients with positive and negative family history of stroke $\left(x^{2}=2.432, p=0.119\right)$.

\section{Influencing Factors Of Stroke}

In this study, 10 factors including sex, age, hypertension duration, smoking habit, drinking habit, physical activity, body mass index, blood pressure grad and family history (including family history of hypertension and stroke) were selected. Cox regression analysis was used to explore the influence of these factors on the occurrence of stroke complications. From the results of Cox regression analysis, these factors have different degrees of influence $(p<0.05)$. The major influencing factors of stroke complications were age $(R R=2.917, p<0.001)$, body mass index $(R R=1.450, p<0.001)$, family history of stroke $(R R=1.386, p<0.001)$, blood pressure grad $(R R=1.148, p<0.001)$, hypertension duration $(R R=$ $1.071, P<0.001)$ and family history of hypertension $(R R=1.051, P<0.001)$. The lower limit value of $R R$ of sex, smoking habit, drinking habit and physical activity were less than 1. See Table 1.

Table 1

The Cox regression analysis results of influencing factors of stroke on hypertension

\begin{tabular}{|lllllllll|}
\hline Variables & B & SE & Wald & df & Sig. & Exp(B) & \multicolumn{2}{c|}{$95 \%$ Cl RR } \\
\cline { 7 - 10 } & & & & & & & Lower & Upper \\
\hline Age & 1.071 & 0.064 & 280.781 & 1 & 0.000 & 2.917 & 2.574 & 3.307 \\
\hline Sex & -0.318 & 0.024 & 180.560 & 1 & 0.000 & 0.728 & 0.695 & 0.762 \\
\hline Smoking habit & 0.066 & 0.010 & 40.361 & 1 & 0.000 & 0.936 & 0.918 & 0.956 \\
\hline Drinking habit & 0.157 & 0.018 & 76.059 & 1 & 0.000 & 0.855 & 0.825 & 0.886 \\
\hline Physical activity & -0.036 & 0.010 & 12.929 & 1 & 0.000 & 0.965 & 0.946 & 0.984 \\
\hline Family history of stroke & 0.326 & 0.048 & 45.422 & 1 & 0.000 & 1.386 & 1.260 & 1.524 \\
\hline Family history of HP & 0.050 & 0.021 & 5.887 & 1 & 0.015 & 1.051 & 1.010 & 1.094 \\
\hline hypertension duration & 0.068 & 0.001 & 4495.326 & 1 & 0.000 & 1.071 & 1.069 & 1.073 \\
\hline Body mass index & 0.372 & 0.097 & 14.825 & 1 & 0.000 & 1.450 & 1.200 & 1.753 \\
\hline Blood pressure grad & 0.138 & 0.013 & 105.190 & 1 & 0.000 & 1.148 & 1.118 & 1.178 \\
\hline
\end{tabular}

\section{Discussion}


The main harm of hypertension lies in its complications. In the incidence spectrum of complications in hypertension patients, the constituent ratio from high to low was cerebrovascular diseases $(49.61 \%)$, diabetes mellitus (34.94\%), heart damage (13.93\%), kidney diseases $(1.04 \%)$, fundus damage $(0.30 \%)$ and peripheral vascular diseases $(0.04 \%)$. Cardiovascular and cerebrovascular diseases were the main complications in patients with hypertension in community [14]. In this study, the cumulative incidence rate of stroke in hypertensive patients was $6.62 \%$, the male was $6.87 \%$ and the female was $6.37 \%$.. The incidence rate of stroke in hypertensive patients was much higher than that incidence rate in general population $[1,3,15]$. Therefore, It's an important link in community stroke prevention and control that strengthen the blood pressure control of patients with hypertension and prevent the occurrence of stroke in patients with hypertension. What needs to be explained here again is that Jiading District government began to implement the free medication policy for hypertension patients with rural medical insurance as early as around 2000. Later, due to the cancellation of rural household registration and the implementation of urban population management policy in Shanghai, the free medication policy gradually faded out, but it is still open to poor households. The vast majority of patients were taking antihypertensive drugs in followed-up hypertension patients in Jiading Shanghai, only a few patients were not willing to accept drug treatment. Assuming these patients did not receive antihypertensive treatment, the incidence of stroke might be higher.

At present, there are many reports on the risk probability analysis or prediction of stroke with hypertension, and the methods used include Logistic Regression analysis, Framinghan evaluation method and ESRs score method [16-19]. The results of different methods are different. Kaplan-Meier method was used in this study, which is also called product limit method. It is not only suitable for small sample data, but also suitable for large sample data. In addition, this method can take into account the objects exiting the observation queue or incomplete censored data, so it is more applicable. Through the analysis of long-term observation data, it was found that with the extension of hypertension years, the cumulative risk probability of stroke in hypertension patients would continue to increase, and the increasing distance was not equidistant. The total cumulative occurred probability of stroke in hypertension patients was 0.789 (78.9\%), and male was 0.910 (91.0\%), female was 0.707 (70.7\%). Male was higher than female. The observation results remind us that hypertension patients must control their blood pressure as soon as possible. With the extension of the course of disease, the risk of stroke will increase, and finally reach a very high risk probability, especially male patients.

The risk occurred probability of stroke in hypertension patients was not fixed during the whole hypertension years, but fluctuates. There were 4 onset peaks, which were in 8 years (peak value 0.042 , $4.2 \%$ ), 15 years (peak value $0.140,14.0 \%$ ), 22 years (peak value $0.060,6.0 \%$ ) and 26 years (peak value $0.139,13.9 \%$ ). Why did the risk probability of stroke in hypertension patients show four different peaks instead of one-way change, it might be the result of long-term conflict between antihypertensive treatment and vascular damage accumulation in hypertension patients, and it might be accompanied by other reasons, which needs further and more study. It is difficult to observe the dynamic changes of hypertension patients in the natural state in practical work based on medical ethics. The highest risk probability of male patients was in 26 years (peak value $0.231,23.1 \%$ ), and the second peak was in 15 
years (peak value $0.156,15.6 \%$ ). The highest risk of female patients was in 15 years (peak value 0.129 , $12.9 \%$ ), and the second peak was in 26 years (peak value $0.087,8.7 \%$ ). If hypertensive patients did not receive antihypertensive treatment, the risk probability of stroke might be higher, and the peak time point might be more advanced.

Stroke is a group of cerebrovascular circulation disorders caused by various reasons, manifested as focal neurological deficit, and even accompanied by disturbance of consciousness. Because of its sudden onset, it is also called cerebrovascular accident. There were many risk factors for stroke, such as age, heredity, hypertension, heart disease, arrhythmia, diabetes, hyperlipidemia, smoking, drinking, obesity, high salt, high animal oil diet, excessive physical activity, etc. [9, 20-27]. The risk of stroke was higher in the hypertension and diabetes population. From the results of Cox regression analysis, these factors have different degrees of influence $(p<0.05)$. According to the results of the long-term observation of registered hypertension patient, the major influencing factors of stroke complications were age $(\mathrm{RR}=$ $2.917)$, body mass index $(R R=1.450)$, family history of stroke $(R R=1.386)$, blood pressure grad $(R R=$ 1.148$,$) , hypertension duration (R R=1.071)$ and family history of hypertension $(R R=1.051)$. These results were consistent with the above literature reports. The influence of sex, smoking habit, drinking habit and physical activity on stroke complications was statistically significant, the lower limit value of RR of were less than 1. This also means that the direction of action of these factors was protective factors or opposite effect. The reason might be affected by the assignment of data classification, for example, male was assigned as 1 and female was assigned as 2 in sex classification, but the risk of male was actually high than that of female. It might also be caused by other factors. This needs further study, but it is undeniable that sex, smoking habit, drinking habit and physical activity are the influencing factors of stroke, which must be paid attention to in community prevention and control.

In short, hypertension patients were prone to stroke, and the risk probability would continue to disproportionately increase during observation, and the total cumulative risk probability could finally reach $78.9 \%$. Male was higher than female. Age, BMI, family history (stroke and HP), blood pressure grad and duration of HP were related to the occurrence of stroke complications. Early control of patients' blood pressure and prevention and control of hypertension related risk factors is an important part and means of community stroke prevention and control.

\section{Abbreviations}

HP

hypertension; BP:blood pressure; SBP:systolic blood pressure; DBP:diastolic blood pressure; BMl:Body mass index; RR:Relative risk; Cl:Confidence interval.

\section{Declarations}

This study was approved by Science and Technology Commission, Health Commission and CDC in Jiading district in Shanghai. All methods were carried out in accordance with relevant guidelines and 
regulations.

\section{Acknowledgements}

Not applicable

\section{Authors' contributions}

All authors have read and approved the manuscript. An-le Li: substantial contributions to the conception and design of the study, analysis of the data冈interpretation of data and the drafting and revision of the paper. Shuai Zhu: substantial contributions to the data acquisition, merging and sorting. Ying Qi, Zhi-hao Hu, Xue-jin Xu,Ya-wei Wang, Xin-zhi Qian: substantial contributions to the data acquisition and data quality control. Final approval of manuscript: All authors.

\section{Funding}

This study was funded by Research project of Natural Science Foundation of Jiading district in Shanghai (JDKW-2020-0036). The funder played no role in the design; conception; data collection, interpretation, and analysis; drafting; or any other process in this paper.

\section{Availability of data and materials}

The data that support the findings of this study are available from the Hypertension Follow-up Management System database in Jiading district in Shanghai, but restrictions apply regarding the availability of these data, which were used under license for the current study and thus are not publicly available. The data are, however, available from the authors upon reasonable request and with permission of the Jiading district health committee in Shanghai.

\section{Ethics approval and consent to participate}

Ethical approval was granted by Jiading district center for disease control and prevention research ethics committee. All subjects gave informed consent to participate in the study, they would like to participate in registry and manage and answer all the related questions in the follow-up.

\section{Consent for publication}

Not applicable.

\section{Competing interests}

The authors declare that they have no competing interests.

\section{References}


1. Ma Lin, Chao Baohua, Cao Lei, et al. Epidemic trend and characteristics of stroke in China from 2007 to 2017 [J]. Chinese Journal of cerebrovascular diseases (Electronic Edition), 2020, 5(14): 253-258.

2. Lozano R, Naghavi M, Foreman K, et al. Global and regional mortality from 235 causes of death for 20 age groups in 1990 and 2010: a systematic analysis for the Global Burden of Disease Study 2010 [J]. Lancet, 2012, 380(9859): 2095-2128.

3. Wang Longde, Liu Jianmin, Yang Yi, et al. The prevention and treatment of stroke in China still faces great challenges - Summary of China stroke prevention and treatment report 2018 [J]. Chinese Journal of circulation, 2019, 34(2): 105-119.

4. Huo Y, Li J, Qin X, et al. Efficacy of folic acid therapy in primary prevention of stroke among adults with hypertension in China: the CSPPT randomized clinical trial [J]. JAMA, 2015, 313(13): 13251335.

5. Hu L, Huang X, Zhou W, et al. Associations between resting heart rate, hypertension, and stroke: A population based cross-sectional study [J]. J Clin Hypertens. 2019, 21(5): 589-597.

6. Huo Y, Li J, Qin X, et al. Efficacy of folic acid therapy in primary prevention of stroke among adults with hypertension in China: the CSPPT randomized clinical trial [J]. JAMA, 2015, 313(13): 325-1335.

7. Meschia JF, Bushnell C, Boden-Albala B, et al. Guidelines for the primary prevention of stroke: a statement for healthcare professionals from the American Heart Association/American Stroke Association [J]. Stroke, 2014, 45(12): 3754-3832.

8. An-le Li, Shuai Zhu, Zhi-Hao Hu, et al. The distribution and epidemic characteristics of cerebrovascular disease in followed-up hypertension patients [J]. Sci Rep. 2021, 11(1):9366. doi: 10.1038/s41598-021-88127-5.

9. Neurology branch of Chinese Medical Association, cerebrovascular disease group of Neurology branch of Chinese Medical Association. Chinese guideline for primary prevention of cerebrovascular disease 2019 [J]. Chinese Journal of Neurology. 2019, (09): 684-709.

10. Cerebrovascular disease group, neurology branch, Chinese Medical Association. Chinese guideline for diagnosis and treatment of acute ischemic stroke 2010 [J]. General practice in China. 2011, 14(35): 4013-4017.

11. Peng B, Wu B. Chinese guideline for diagnosis and treatment of acute ischemic stroke 2018 [J]. Chinese Journal of Neurology. 2018, 51(09): 666-682.

12. Liu LS. Chinese guidelines for prevention and treatment of hypertension 2010. Chinese Journal of hypertension [J]. 2011, 19(08): 701-743.

13. China Association of hypertension, cardiovascular branch of Chinese Medical Association, etc. China hypertension prevention and control guide (revised version 2018) [J]. Chinese Journal of cardiovascular disease. 2019, 24(01): 24-56.

14. Li AL, Shao YQ, Yuan H, Zhang YY. Incidence spectrum of hypertension in Jiading District of Shanghai [J]. Prevention and control of chronic diseases in China. 2016,24(11): 870-872.

15. Qi W, Ma J, Guan T, Zhao D, Abu-Hanna A, Schut M, Chao B, et al. Risk Factors for Incident Stroke and Its Subtypes in China: A Prospective Study. J Am Heart Assoc. 2020;9(21):e016352. doi: 
10.1161/JAHA.120.016352. Epub 2020 Oct 26.

16. Jiang HM. The value of Framingham stroke risk score combined with RDW in predicting the longterm stroke risk of patients with H-type hypertension [J]. Sichuan Medical University. 2020, 41(01):56-61.

17. Tang X, Zhang DD, Liu XF, Liu QP, et al. Application of China par stroke model in predicting stroke risk in northern rural population [J]. Journal of Peking University (Medical Edition). 2020, 52(3): 444-450.

18. Zeng J, Liu Q, Lin AH. Analysis of influencing factors and risk prediction of ischemic stroke among middle-aged and elderly people in Guangzhou community [J]. Journal of Sun Yat sen University: Medical Science Edition. 2016,(4):614-620.

19. Fu Q, Huang YJ, Jiang SZ, Li XD. Ten year risk probability analysis of stroke in hypertension patients [J]. Chinese Journal of cerebrovascular diseases (Electronic Edition). 2012,(6)5:22-26.

20. Wang CY, Cao LM, Shi J, Li X, Hu FL, Ma JP, et al. A prospective cohort study on blood pressure control and risk of ischemic stroke in patients with hypertension [J]. Zhonghua Yu Fang Yi Xue Za Zhi. 2020; 54(7):737-741. doi: 10.3760/cma.j. cn112150-20191225-00958

21. An-le Li, Qian Peng, Yue-Qin Shao, Xiang Fang, Yi-Ying Zhang. The interaction on hypertension between family history and diabetes and other risk factors [J]. Sci Rep. 2021;11(1):4716. doi: 10.1038/s41598-021-83589-z.

22. Zhou F, Hou D, Wang Y, Yu D. Evaluation of H-type hypertension prevalence and its influence on the risk of increased carotid intima-media thickness among a high-risk stroke population in Hainan Province, China. Medicine (Baltimore). 2020;99(35):e21953. doi: 10.1097/MD.0000000000021953.

23. Li AL, Peng Q, Shao YQ, Fang X, Zhang YY. The effect of body mass index and its interaction with family history on hypertension: A case-control study. Clin. Hypertens. 2019;25(6):1-8.

24. Dai J, Wang X, zhu XY, Yang YJ, zheng KW. A study on the influencing factors of stroke in China based on multi-layer Logit model [J]. Chinese Health Statistics. 2020, 37(05):708-711.

25. 25..Du QM, Cao SH, Su M, et al. Risk factors and prognosis of stroke patients of different ages and genders [J]. Chinese Journal of gerontology. 2021, 41(07): 1374-1377.

26. Wang YJ. Secondary prevention of ischemic stroke [J]. Chinese Journal of Neurology. 2021, 54(02): 139-148.

27. Guo ZG, Huang JY, Meng RW, et al. Prevalence of Hyperhomocysteine and its correlation with stroke risk in community residents over 40 years old in Shenzhen [J]. Chinese Journal of health management. 2021, 15(01): 37-43.

\section{Figures}




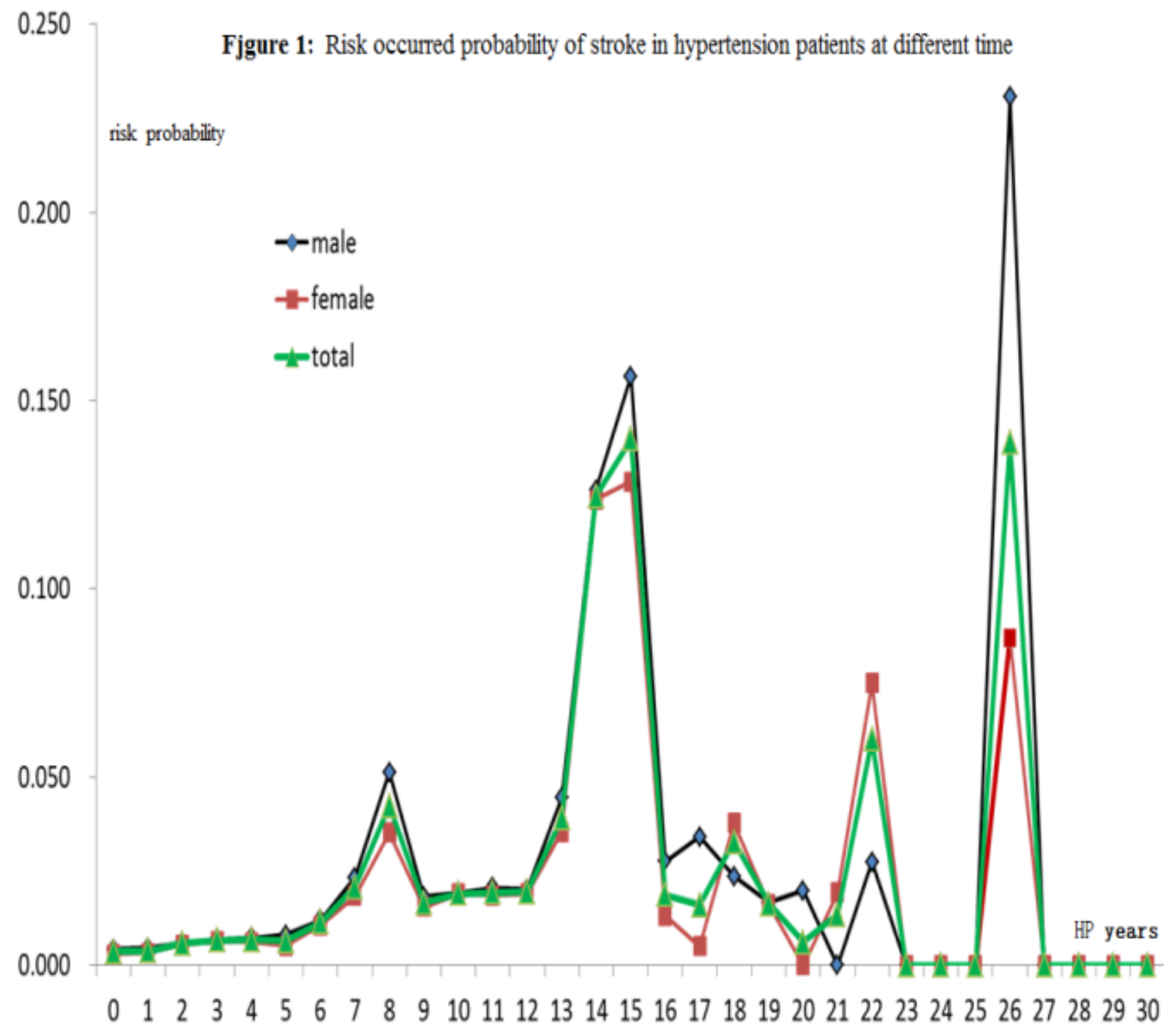

Figure 1

See image above for figure legend 
Figure 2: Sex and hazard of stroke

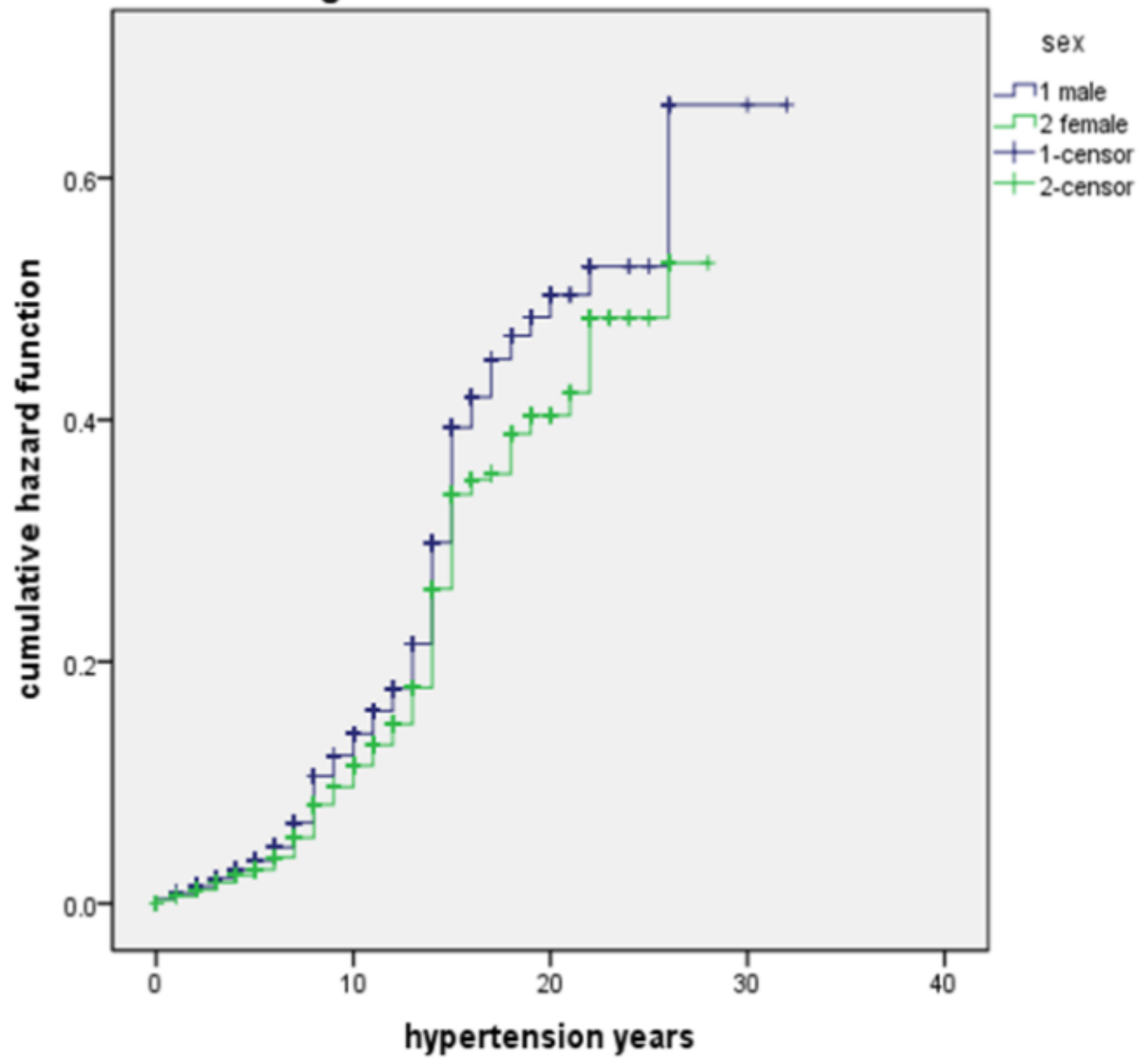

Figure 2

See image above for figure legend 
Figure 3: Blood pressure and hazard of stroke

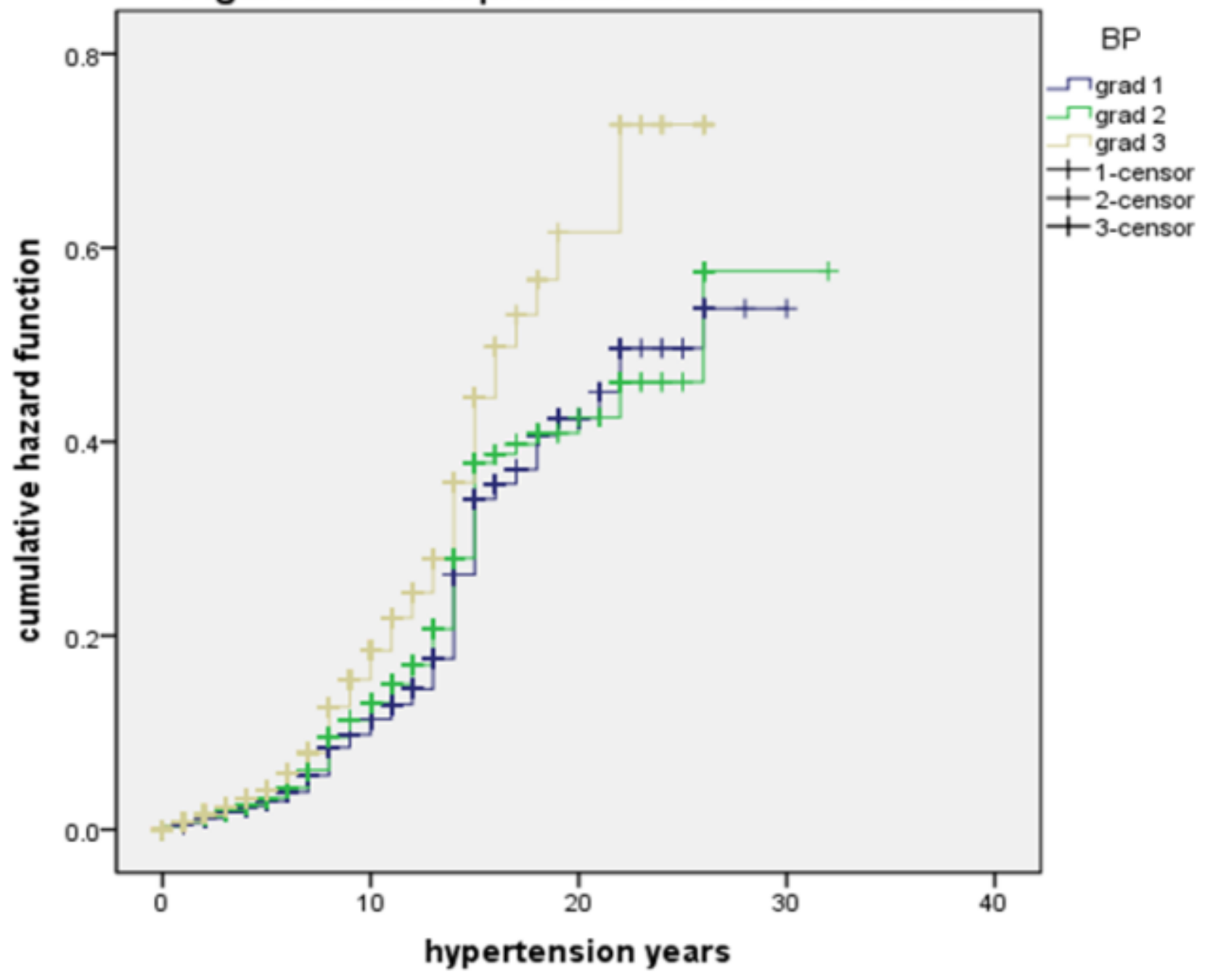

Figure 3

See image above for figure legend 
Figure4: Body mass index and hazard of stroke

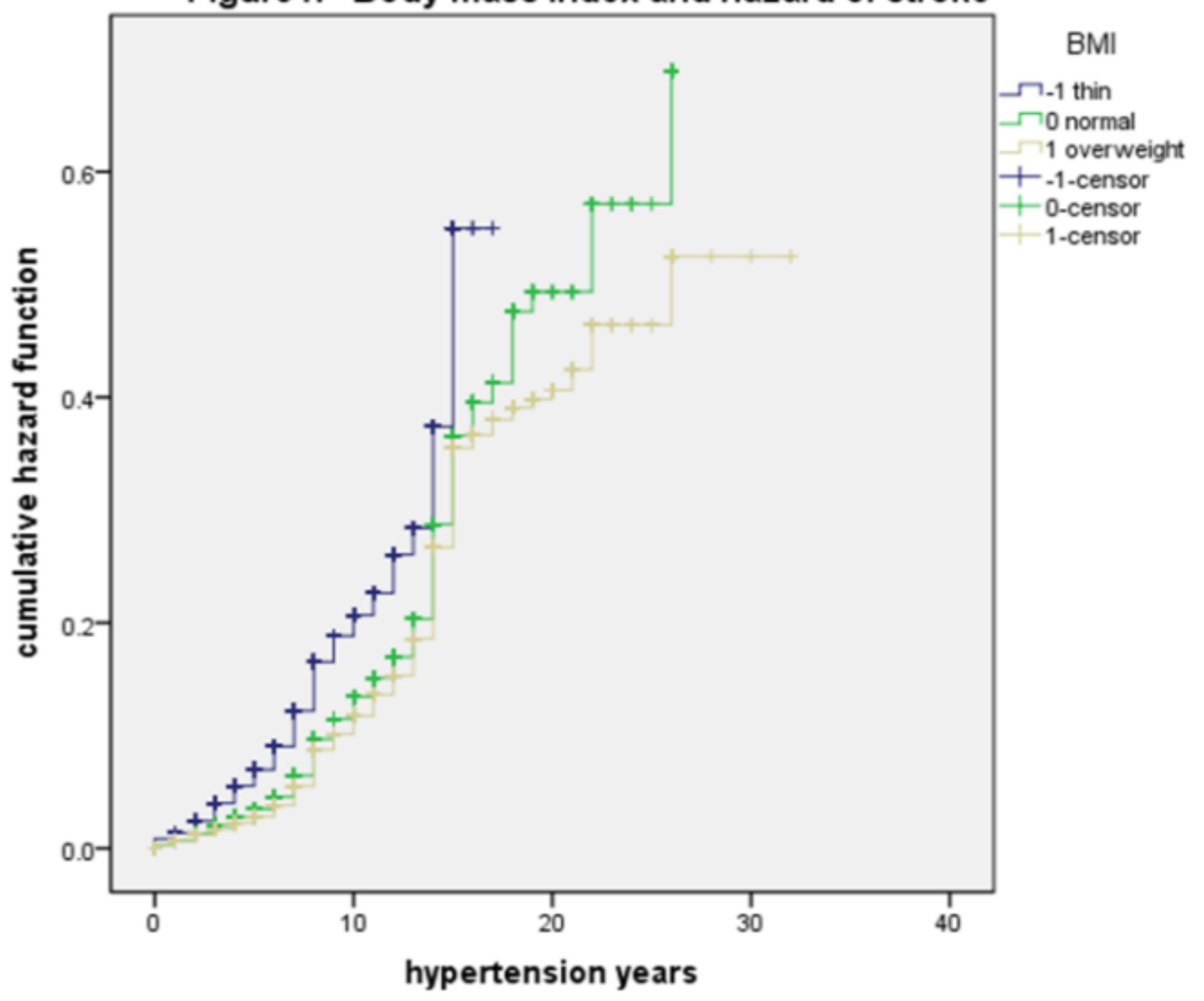

Figure 4

See image above for figure legend 


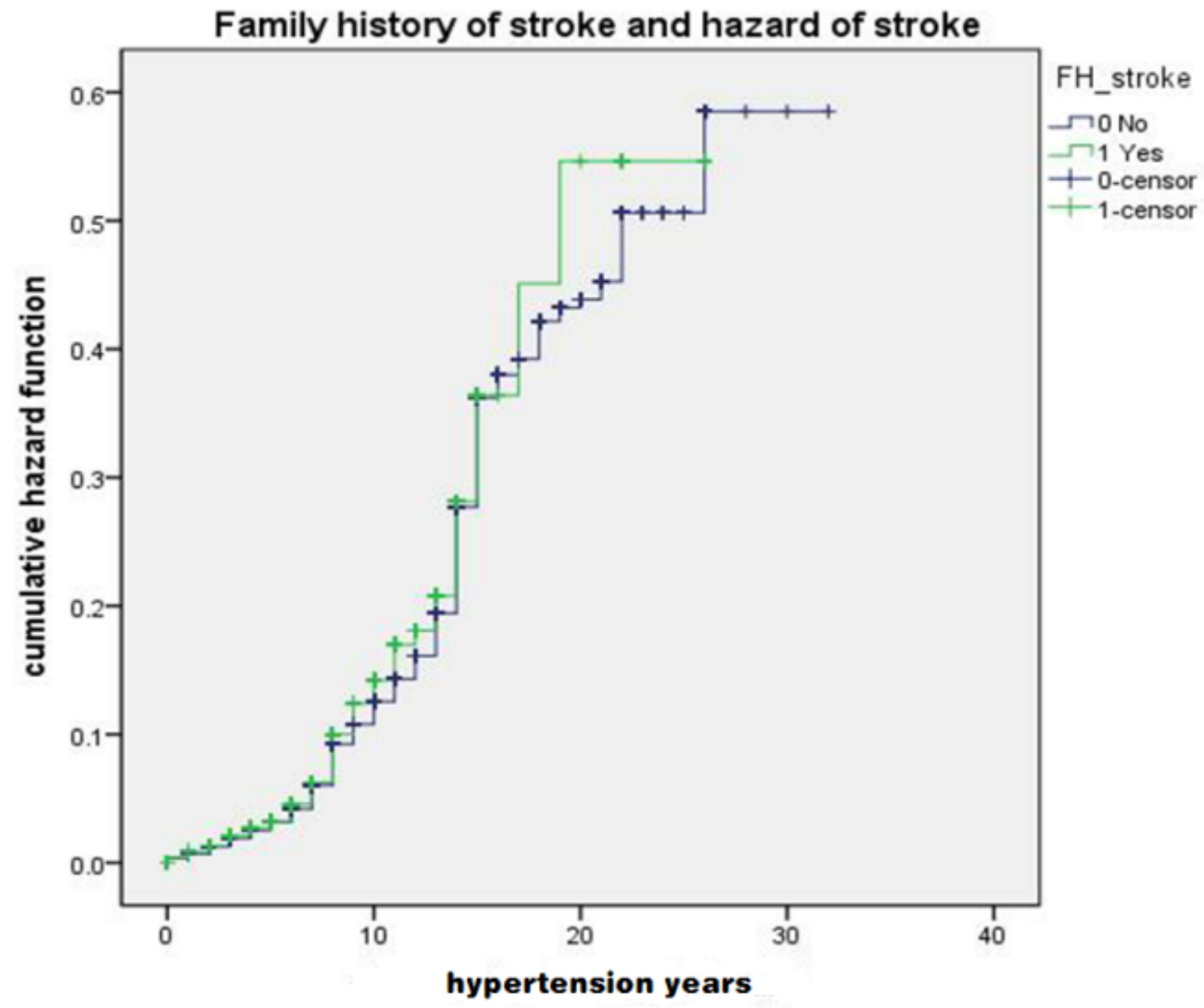

Figure 5

See image above for figure legend 